\title{
The Yarkovsky-driven origin of near-Earth asteroids
}

\author{
A. Morbidelli ${ }^{\mathrm{a}, *}$ and D. Vokrouhlickýb \\ a Observatoire de la Côte d'Azur, Boulevard de l'observatoire, B.P. 4229, 06304 Nice cedex 4, France \\ ${ }^{\mathrm{b}}$ Institute of Astronomy, Charles University, Prague, Czech Republic
}

Received 23 September 2002; revised 6 January 2003

\begin{abstract}
We investigate the relevance of the Yarkovsky effect for the origin of kilometer and multikilometer near-Earth asteroids (NEAs). The Yarkovsky effect causes a slow migration in semimajor axis of main belt asteroids, some of which are therefore captured into powerful resonances and transported to the NEA space. With an innovative simulation scheme, we determine that in the current steady-state situation 100-160 bodies with $H<18$ (roughly larger than $1 \mathrm{~km}$ ) enter the $3 / 1$ resonance per million years and $40-60$ enter the $\nu_{6}$ resonance. The ranges are due to uncertainties on relevant simulation parameters such as the time scales for collisional disruption and reorientation, their size dependence, and the strength of the Yarkovsky and YORP effects. These flux rates to the resonances are consistent with those independently derived by Bottke et al. (2002, Icarus 156, 399-433) with considerations based only on the NEA orbital distribution and dynamical lifetime. Our results have been obtained assuming that the main belt contains 1,300,000 asteroids with $H<18$ and linearly scale with this number. Assuming that the cumulative magnitude distribution of main belt asteroids is $N(<H) \propto 10^{\gamma^{\prime} H}$ with $\gamma^{\prime}=0.25$ in the 15.5 $<H<18$ range (consistent with the results of the SDSS survey), we obtain that the bodies captured into the resonances should have a similar magnitude distribution, but with exponent coefficient $\gamma=0.33-0.40$. The lowest value is obtained taking into account the YORP effect, while higher values correspond to a weakened YORP or to YORP-less cases. These values of $\gamma$ are all compatible with the debiased magnitude distributions of the NEAs according to Rabinowitz et al. (2000, Nature 403, 165-166), Bottke et al. (2000b, Science 288, 2190-2194), and Stuart (2001, Science 294, 1691-1693). Hence the Yarkovsky and YORP effects allow us to understand why the magnitude distribution of NEAs is only moderately steeper than that of the main belt population. The steepest main belt distribution that would still be compatible with the NEA distribution has exponent coefficient $\gamma^{\prime} \sim 0.3$.
\end{abstract}

(C) 2003 Elsevier Science (USA). All rights reserved.

Keywords: Asteroids; dynamics

\section{Introduction}

The Solar System small bodies with perihelion distances $q \leq 1.3 \mathrm{AU}$ and aphelion distances $Q \geq 0.983 \mathrm{AU}$ are usually called near-Earth asteroids (NEAs). Although originally believed to be dormant cometary nuclei, the prevailing opinion starting from the early 1980s (Wetherill, 1979, Wisdom, 1983) is that these bodies are in fact former main belt asteroids, which escaped from the main belt by resonant effects, achieving planet-crossing orbits (see Morbidelli et al. (2002a) for a review). Several resonances have been identified that are able to pump the eccentricity of asteroids

* Corresponding author. Fax: +33-492003033.

E-mail address: morby@obs-nice.fr (A. Morbidelli). up to planet-crossing values. It is suitable to classify them into "powerful resonances" and "diffusive resonances" (Morbidelli, 2002; Nesvorný et al., 2003). The former can be effectively distinguished from the latter by the existence of associated gaps in the main belt asteroid distribution. The most notable powerful resonances are the $\nu_{6}$ secular resonance, at the inner edge of the asteroid belt, and the 3/1 mean-motion resonance with Jupiter at $2.5 \mathrm{AU}$ (but also the $5 / 2$ and 2/1 resonances at 2.8 and 3.2 AU). The diffusive resonances are so numerous that they cannot be effectively enumerated and they can slowly drive a significant number of asteroids toward the Mars-crossing zone (Migliorini et al., 1998; Morbidelli and Nesvorný, 1999).

If the resonances were not continuously resupplied by new asteroids, they would become completely depleted of 
bodies and NEA production would stop. In this case, in several tens of millions of years the NEAs would disappear. But this has never happened during the history of the Solar System, as witnessed by the terrestrial and lunar crater records (Grieve and Shoemaker, 1994, Neukum and Ivanov, 1994), which show that the NEA population has been roughly constant over the last 3.5 Gyr. The classical scenario developed by Wetherill $(1979,1985,1987,1988$; see also Greenberg and Nolan, 1989; Farinella et al., 1993 and Rabinowitz, 1997) is that the collisional activity in the main belt, which breaks large asteroids, continuously injects a considerable number of new bodies into the resonances. In this manner, the NEA population is kept in steady state.

In the 1990s, the availability of fast computers and new integration algorithms allowed the simulation of the longterm dynamical evolution of a statistically significant number of real or synthetic NEAs (Farinella et al., 1994; Migliorini et al., 1997; Gladman et al., 1997, 2000). It became rapidly evident that the dynamical lifetime of NEAs is only a few million years, more than an order of magnitude shorter than previously computed by Monte Carlo simulations that accounted only for the effect of close encounters with the planets and not for resonant perturbations. This new result implies that, to keep the NEA population in steady state, the rates at which resonances are resupplied with asteroids must be much higher than previously believed. More recently, Bottke et al. (2002) established the relative contribution to the NEA population of the most important resonant sources. Assuming that the NEA population is in steady state, they estimated that about 220 asteroids brighter than absolute magnitude $H=18$ (roughly larger than $1 \mathrm{~km}$ in diameter) escape from the inner main belt $(a<2.8 \mathrm{AU})$ every million years; of these, $100 \pm 50$ should come through the $3 / 1$ resonance, $55 \pm 18$ through the $\nu_{6}$ resonance, and $65 \pm 15$ through the network of diffusive resonances in the 2.1- to 2.8-AU region. To date, these are the best estimates of the resupply rate of the NEA source resonances.

Zappalà et al. (2002) have searched for all main belt asteroids which, upon collisions, could inject multikilometer bodies into the NEA source resonances. They found a relatively small number of candidates, which made them conclude that collisions are unlikely to produce the required number of NEAs larger than $1 \mathrm{~km}$. Taking into account that they considered optimistic ejection velocity fields, unsupported by smooth particle hydrodynamics simulations (Love and Ahrens, 1996; Benz and Asphaug, 1999; Michel et al., 2001), this result puts in serious doubts the classical scenario on the collisional origin of NEAs.

There are two additional indications that the NEAs should not be fresh collisional fragments. The first comes from their shallow magnitude distribution. Several works (Rabinowitz et al., 2000; Bottke et al., 2000b; Stuart, 2001) agree that the cumulative $H$ distribution of the NEAs in the $14<H<22$ range has the form

$$
N(<H) \propto 10^{\gamma H},
$$

with $\gamma=0.35$ to 0.39 . If the NEAs were fragments from catastrophic breakups, they would presumably have a much steeper magnitude distribution, as predicted by hydrocode simulations (Michel et al., 2001) and observed in asteroid families ( $\gamma>0.5$; Tanga et al., 1999; Campo Bagatin et al., 2002). The second indication comes from the heavily cratered surface of Eros, revealed by the NEAR spacecraft. The large number of craters suggests that the physical age of Eros (2 Gyr; Chapman et al., 2002) is much greater than its probable dynamical lifetime as a NEA (few tens of millions of years; Michel et al., 1996), which implies the asteroid was not a fresh collisional fragment at the time of its insertion into the resonance that transported it to the NEA space.

From these considerations, in their review on the current state of art, Morbidelli et al. (2002a) concluded that the Yarkovsky effect might be ultimately responsible for the delivery of a sufficiently large number of kilometer-sized asteroids to the NEA source resonances. The Yarkovsky effect is a weak recoil force due to the thermal reemission of rotating asteroids, which causes kilometer-sized bodies to slowly drift in semimajor axis (Farinella and Vokrouhlický, 1999; see Bottke et al., 2003, for a review). The drift rate is estimated to be $\sim 10^{-4} \mathrm{AU} \mathrm{Myr}^{-1}$ for $1-\mathrm{km}$ bodies and to be roughly inversely proportional to the body's size. The same phenomenon has already been shown to work for the delivery of meteorite precursor bodies to the same resonances and explains the long cosmic ray exposure ages of meteorites (Vokrouhlický and Farinella, 2000; Bottke et al., 2000a).

The purpose of this paper is to verify the role of the Yarkovsky effect for the origin of NEAs. Our goal is twofold: (i) to quantify the rates at which bodies with $H<18$ are transported by the Yarkovsky effect into the 3/1 and $\nu_{6}$ resonance and (ii) to quantify the cumulative magnitude distribution of the bodies that enter these resonances. We do this with a combination of numerical integration of the Yarkovsky equations and Monte Carlo techniques, in which the bodies drift in semimajor axis under the Yarkovsky effect at constant eccentricity and inclination, statistically undergoing spin axis reorientations and collisional disruptions. The results are compared with the supply rates for the $3 / 1$ and $\nu_{6}$ resonances estimated by Bottke et al. (2002) and with the observed cumulative magnitude distribution of the NEA population. Notice that we do not investigate the rates at which the bodies are supplied to the diffusive resonances, because this would require full $\mathrm{N}$-body simulations accounting for the gravitational perturbations exerted by the planets, as in Bottke et al. (2001).

This work requires a quite complex and articulated modeling effort. We start in Section 2 by constructing a simple model of the orbital and absolute magnitude distribution of main belt asteroids, based on current observations. In Section 3 we discuss how we can define the boundaries of the 


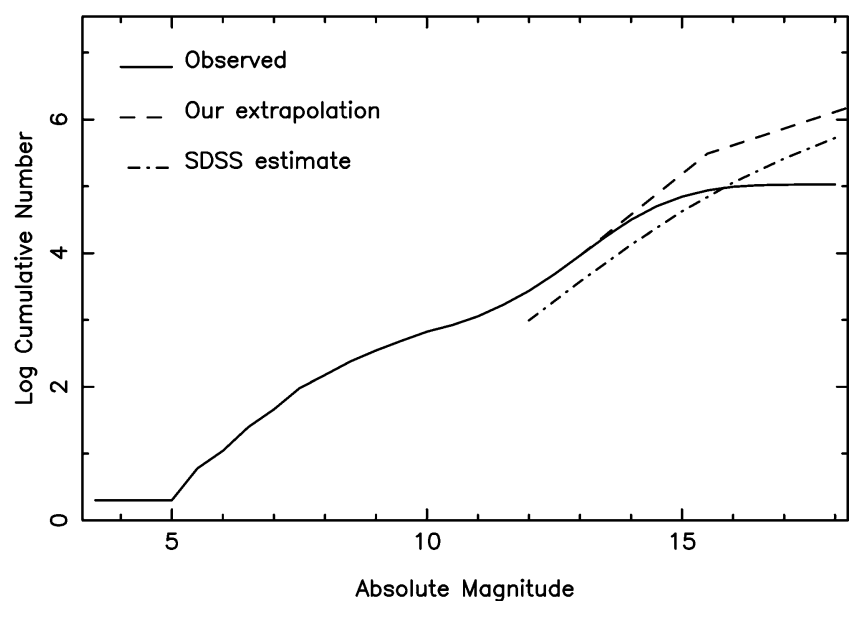

Fig. 1. Cumulative magnitude distribution of main belt asteroids. The solid curve is the observed distribution as given by the Astorb catalog. The dash-dotted curve represents the population predicted by the SDSS survey team (Ivezić et al., 2001). Notice the strong disagreement with the observed distribution, discussed in the text. The dashed lines show our estimate of the population, obtained by extrapolating the observed distribution using a broken power law with slopes very similar to the asymptotic values determined by SDSS.

resonances, through which the effective flux of asteroids can be measured. Section 4 will be devoted to modeling the Yarkovsky and the YORP effects. The latter is the analog of the Yarkovsky effect but acts on the spin motion of the asteroids (Rubincam, 2000, Vokrouhlický and Čapek, 2002). In Section 5 we will describe how we can simulate a steady-state process, where asteroids are continuously produced and disrupted in the main belt with some characteristic (size-dependent) collisional lifetime, while the Yarkovsky effect delivers to the resonances those bodies which are formed closest to the resonance boundaries. In Section 6 we analyze the resulting fluxes of bodies into the $3 / 1$ and $\nu_{6}$ resonances and their cumulative magnitude distribution. We also investigate how these results change with the values of the parameters of the model. Conclusions and discussion will close the work in Section 7.

\section{A simple main belt asteroid distribution model}

To estimate the flux of main belt asteroids into the NEA source resonances we need to have a model of the orbital and absolute magnitude distribution of the main belt population. Fig. 1 shows the observed cumulative $H$ distribution of main belt asteroids (solid curve). Unfortunately, the known population is severely incomplete for $H>13-14$, so that beyond this threshold we need to make an educated guess on what should be the real distribution.

An important contribution is provided by the results of the recent SDSS survey which, from the detection of 13,000 asteroids over $500 \mathrm{deg}^{2}$ of searched sky, concluded that the cumulative magnitude distribution can be approximated by a broken exponential law of type $N(<H) \propto 10^{\gamma^{\prime} H}$, with $\gamma^{\prime}$ $=0.6$ for $12<H<15.5$ and $\gamma^{\prime}=0.26$ for $15.5<H<19$ (Ivezić et al., 2001). This broken exponential law can be approximated by an analytic function which asymptotically approaches the two slopes, represented by the dash-dotted curve in Fig. 1. By estimating which fraction of the main belt population should have crossed the survey field, Ivezić et al. concluded that there should be 530,000 asteroids with $H<18$ and 68,000 with $H<15.5$. Notice however that Jedicke and Metcalfe (1998), from the sample of the main belt population detected by the Spacewatch survey, estimated 120,000 bodies with $H<15.5$, while the number with $H<18$ is not explicitely reported.

A problem with the estimate provided by the SDSS team is that, as evident in Fig. 1, the number of predicted bodies is smaller than the observed number, for $H<16$. To explain this apparent paradox, the SDSS team (Jurić et al., 2002) argued for a systematic error in the absolute magnitudes reported in the Astorb catalog (see ftp://ftp.lowell.edu/pub/ elgb/astorb.html) and, to a lesser extent, in the database of the Minor Planet Center (see http://cfa-ftp.harvard.edu/pub/ MPCORB). In essence, Jurić et al. claimed that the asteroids are reported in these catalogs with a value of $H$ that is, on average, 0.5 magnitudes brighter than the "real" one. This means that the solid curve in Fig. 1 should be shifted to the right by 0.5 magnitudes, which would make it coincide quite accurately with the SDSS estimated distribution. On the other hand, we note in Fig. 1 that the disagreement between the observed and SDSS distributions also concerns fairly bright bodies with $H=12-13$, while the discrepancies in the cataloged magnitudes reported by Jurić et al. concern essentially only the bodies with apparent magnitude $V>15$; the latter, at opposition at $2 \mathrm{AU}$, corresponds to $H$ $>13.5$. Also, the disagreement between the SDSS and the Jedicke and Metcalfe estimates is likely not explained by an error in the reported absolute magnitudes, because Jedicke and Metcalfe used exclusively Spacewatch data, whose photometry is "notoriously good" (R. Jedicke, private communication, 2002).

At the current state of the art, the situation is rather confused. It might be possible that there is a problem in the conversion factor that the SDSS team uses to convert the number of asteroids detected by their survey into the number of total asteroids in the main belt. If this were the case, the slopes of the magnitude distribution determined by the SDSS should be basically trustworthy, but not the estimate of the total number of main belt asteroids. Following this conjecture, we have extrapolated the observed cumulative distribution using SDSS-like slopes, namely, 0.61 for $13<$ $H<15.5$ and 0.25 for $15.5<H<18$. We remark that the first slope fits very well the observed slope for $H$ in the 12-14 range; the second slope has also been found by Jedicke and Metcalfe (1998). Our extrapolated distribution, shown in Fig. 1 by dashed lines, predicts 1,300,000 bodies with $H<18$. This number is in good agreement with the number of bodies larger than $1 \mathrm{~km}$ predicted by Tedesco 
and Desert (2002) using the observations of the infrared satellite ISO. In the following we will use this model as our nominal population, for the computation of the asteroid flux into resonance. The reader should keep in mind that if the SDSS estimate of the total population eventually turns out to be correct, the fluxes that we obtain should be divided by 2.5 (the ratio between the number of $H<18$ objects in our estimate and in the SDSS estimate).

In practice, we partition the belt into three zones: the inner belt with $a<2.5 \mathrm{AU}$, the central belt with $2.5<a<$ $2.82 \mathrm{AU}$, and the outer belt with $2.82<a<3.3 \mathrm{AU}$ (which is never considered in this work, since it cannot contribute to the flux of objects into the $3 / 1$ or $\nu_{6}$ resonances). In each zone, we extrapolate the observed $H$ distribution using the slopes reported in the previous paragraph. We conclude that the inner belt and the central belt should host respectively 175,000 and 388,000 bodies with $H<18$.

After having assumed an absolute magnitude distribution of the main belt population in each zone, we complete the catalog of the observed asteroids by generating synthetic objects. A nontrivial problem is to decide which orbital distribution should be given to the synthetic objects. The asteroid belt population can be partitioned into several family groups and a background subpopulation (Tedesco et al., 2002). Until recently it was generally accepted that the magnitude distribution of the former is steeper than that of the latter (Zappalà and Cellino, 1996). If this were true, most of the synthetic asteroids should be attributed to the family groups. However, using the latest data and an innovative approach, we have recently shown that the $H$ distribution of asteroid families bends very sharply beyond $H=$ 13, and that for $H>15$ it is background-like or even slightly shallower (Morbidelli et al., 2003). In this case the relative partition of the synthetic asteroids among background and families subpopulations should be roughly similar to the partition of the observed asteroids. This is the attitude that we adopt in this paper.

A second problem concerns the orbital distribution of the asteroids within each family. Cellino et al. (1999) showed that the orbital distribution of family members depends on $H$. The new data, however, show that this is true only for $H$ $\lesssim 13$ (Bottke et al., 2001; see also Fig. 2). Above this threshold, the families typically spread over a region extending between the two nearest powerful resonances, which sharply bound the family. Bottke et al. interpreted this fact as a signature of the dispersion of asteroid families due to the Yarkovsky force. In fact, the drift in semimajor axis induced by the Yarkovsky effect is size dependent: the smaller the bodies, the further they can drift in a given time (the family age). This gives an $\mathrm{H}$-dependent size of the family, or a V-shape structure in a semimajor axis vs $H$ representation (Fig. 2). However, powerful resonances cannot be crossed by bodies slowly drifting in semimajor axis, so that the families cannot extend beyond the nearest gaps on the left and on the right side. As a consequence, the semimajor axis dispersion of the family members fainter

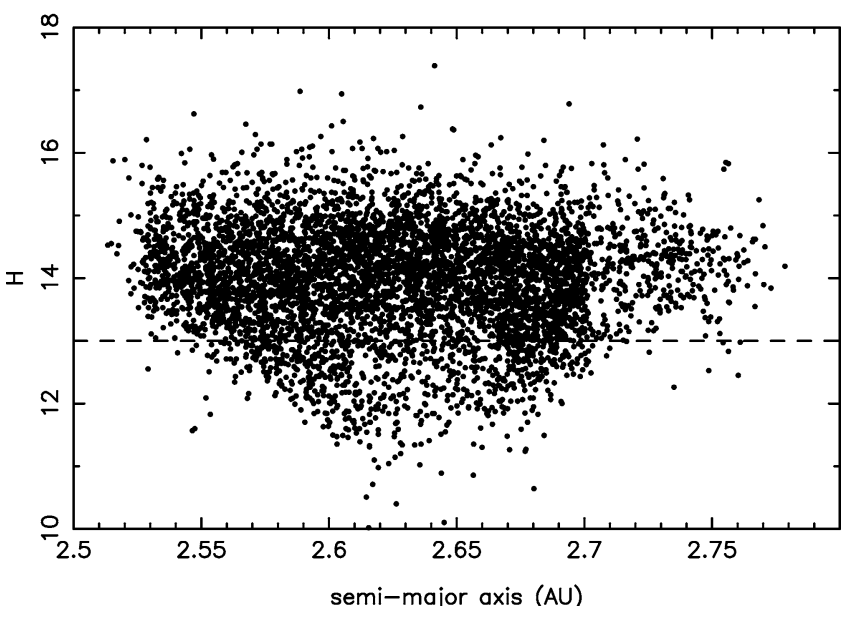

Fig. 2. The observed (a, $H$ ) distribution of the Eunomia family, defined from the new catalog of $\sim 100,000$ proper elements by Milani and Knežević (see Knežević et al., 2003, for a review). The horizontal line at $H=13$ separates the lower region, where the width of the family depends on $H$, from the upper region, where the semimajor axis distribution of the family members is roughly independent of $H$. The same happens for all the major families in the asteroid belt.

than some threshold (typically $H \sim 13-14$ ) is simply limited by the resonance locations, and results independent of $H$.

These considerations on the orbital and magnitude distribution of asteroid families make us conclude that, in first approximation, the orbital distribution of the still unobserved asteroids with $13<H<18$ should be statistically equivalent to that of the observed bodies in the same magnitude range. In doing this, we neglect that there are relative biases favoring the discovery of low inclination and/or small perihelion distance objects over the large inclination and/or large perihelion distance ones. These biases are probably not overwhelming inside each of the three main belt zones defined here, thanks to the limited range of $a$, $q$, and $i$ spanned by the bodies in each zone. This motivated us to partition the belt into these three zones and to neglect the existence of relative biases inside each zone.

In conclusion, to keep the model as simple as possible, in each zone we randomly attribute to the synthetic asteroids the values of proper semimajor axis, eccentricity, and inclinations of the observed asteroids with $H>13$. In doing this, in our model we end up having several objects initially with identical values of semimajor axis, eccentricity, and inclination. This is not a problem, because having different sizes, spin axis orientation, and spin rates, these bodies will drift in semimajor axis at different speeds under the Yarkovsky effect, and thus they will have different evolutions. Size, spin axis orientation, and spin rate are key parameters in the determination of the Yarkovsky force. The assumptions for the latter two are explicitely discussed in Section 4. The sizes are derived from the absolute magnitudes, by assuming that the albedo of the bodies in the inner belt is 0.25 and that the central belt is 0.22 . These values correspond to the mean albedos of the main belt asteroids in the vicinity of the 

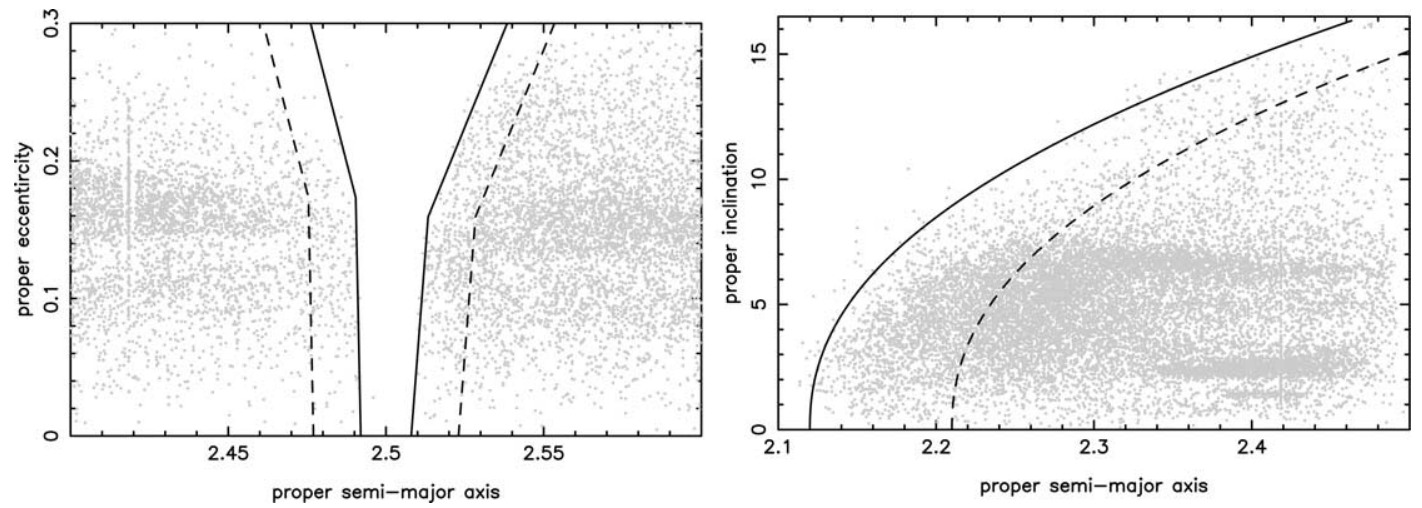

Fig. 3. The distribution of the asteroids with respect to the numerically computed proper elements, in the vicinity of the $3 / 1$ (left panel) and $\nu_{6}$ (right panel) resonances. The continuous curves show the boundaries defined in (2), (3), and (4). The dashed curves show the shifted boundaries used for the computation of the effective asteroid fluxes.

$\nu_{6}$ and 3/1 resonances, respectively (Morbidelli et al., $2002 \mathrm{~b}$ ). We are aware that the bodies close to $2.8 \mathrm{AU}$ should have smaller albedos, on average, but this is irrelevant for our purposes because these asteroids are too far from the $3 / 1$ resonance to contribute to its influx.

Despite all these simplifications, we think that this simple main belt distribution model is enough for our purpose, which is to obtain a first gross estimate of the Yarkovskydriven flux of multikilometer bodies into the NEA source resonances.

\section{The effective boundaries of the $3 / 1$ and $\nu_{6}$ resonances}

To evaluate the flux of asteroids into the $3 / 1$ and $\nu_{6}$ resonances we need to define the boundaries of these resonances and monitor which bodies cross them during the simulation. To define these boundaries, we refer to the catalog of the synthetic proper elements (42,868 bodies; Knežević and Milani, 2000). The proper $a, e, i$ distribution of the bodies in this catalog shows well-defined gaps associated with the 3/1 and $\nu_{6}$ resonances (Fig. 3), whose boundaries can be approximated by the formulas

$$
\begin{aligned}
& a=2.508+\frac{e}{29.615} \text { for } e \leq 0.15936, \\
& a=2.485+\frac{e}{5.615} \text { for } e>0.15936
\end{aligned}
$$

for the right side of the $3 / 1$ resonance;

$$
\begin{aligned}
& a=2.492-\frac{e}{108.85} \text { for } e \leq 0.1734, \\
& a=2.51-\frac{e}{8.85} \text { for } e>0.1734,
\end{aligned}
$$

for the left side of the 3/1 resonance (independent of $i$ ), and

$$
a=2.12+6.003(\sin i)^{2.256}
$$

for the right side of the $\nu_{6}$ resonance (independent of $e$ ). We use the synthetic proper elements, rather than the analytic proper elements, because the latter would show artificially enlarged resonant gaps, since the analytic algorithm may not converge for bodies in the vicinity of the resonances. Similarly, we do not use the osculating orbital elements, because the latter would show artificially shrunk gaps, since the asteroids may temporarily "invade" the gap during the short periodic oscillations of their semimajor axes.

A visual analysis of Fig. 3 suggests that the density of asteroids sharply decays with increasing proximity to the resonant boundaries. This is not an optical illusion. For example, Fig. 4 (left panel) shows the number of asteroids in the synthetic proper element catalog as a function of distance from the right border of the $3 / 1$ resonance. The distance is measured as $a^{\prime}-a$, where $a^{\prime}$ is the proper semimajor axis of the asteroid, and $a$ is given by (3) using for $e$ the proper eccentricity of the asteroid. As one can see, the asteroid density sharply increases up to $\sim 0.015 \mathrm{AU}$ from the resonance boundary, and then is more or less constant over the next $0.025 \mathrm{AU}$. The reason for this partial depletion of the main belt population close to the 3/1 resonance has been recently discussed by Guillens et al. (2002), who showed with numerical integrations that the dynamics in the vicinity of the resonance are weakly chaotic. Consequently, asteroids within 0.01 to $0.02 \mathrm{AU}$ from the resonance borders tend to migrate in proper element space (chaotic diffusion), falling into the resonance on a time scale of $\sim 100$ Myr. This phenomenon is illustrated in Fig. 4 (right panel), which shows the 100-Myr evolution in proper element space of 45 bodies, initially chosen on a regular grid. It is evident that chaotic diffusion can easily deplete the neighborhood of the resonance up to $\sim 0.0075 \mathrm{AU}$ from the resonance border.

Guillens et al. argued that the diffusive neighborhood of the resonance is not completely depleted of asteroids, because new bodies are continuously resupplied by Yarkovsky 

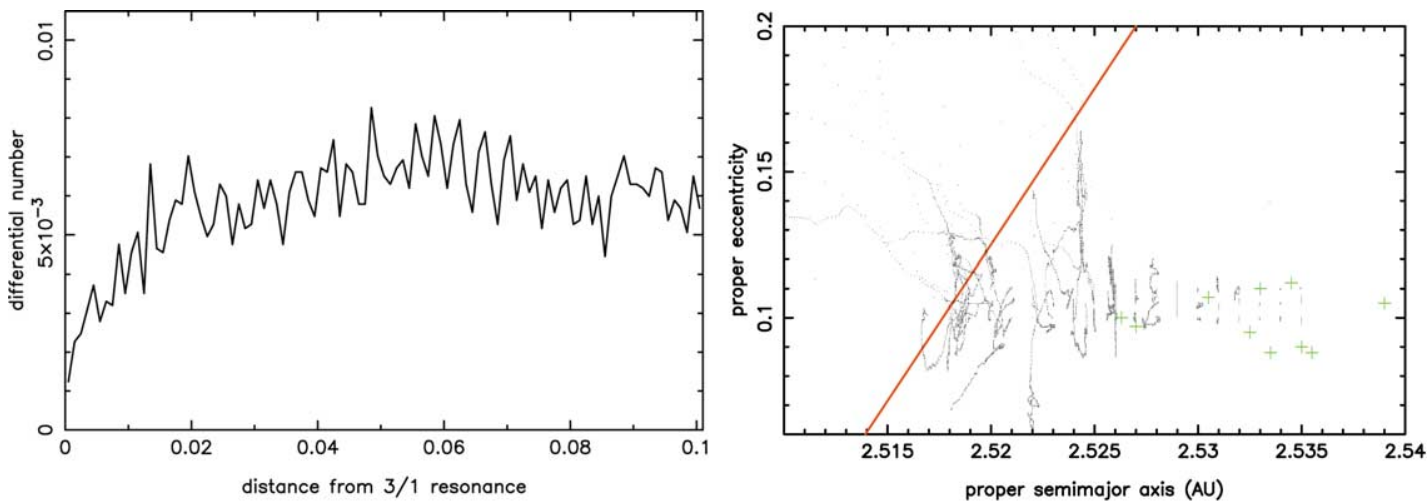

Fig. 4. (Left) The number of asteroids in the synthetic proper elements catalog, as a function of distance from the right border of the 3/1 resonance. Notice the sharp increase of the asteroid density over the first 0.015 AU. (Right) The 100-Myr evolution in the resonant proper element space (see Morbidelli et al., 1995, for a definition of resonant proper elements) of 45 bodies initially chosen on a regular grid in the proper (a,e) plane. Bodies that do not significantly migrate during the integration appear as short vertical segments, while those that do migrate leave a tortuous trace. The slanted line denotes the boundary of the 3/1 resonance, where several migrating bodies eventually enter. The crosses represent some members of the Maria family. The family stops where chaotic diffusion becomes effective.

from the more distant regions of the belt. In this scenario the asteroids drifting in semimajor axis by Yarkovsky enter the diffusive region. Once there, chaotic diffusion becomes improtant, delivering the asteroids to the resonance on a time scale comparable or shorter than the one that would have been necessary if the Yarkovsky effect had to push them all the way to the resonance border (kilometer-sized bodies take $\sim 100 \mathrm{Myr}$ to cross the 0.015 -AU-wide region, on average). The sharply dropping density of asteroids observed in the resonance vicinity is the result of this process. As a further confirmation, Guillens et al. also pointed out that the ratio between small and large asteroids in the diffusive region is larger than it is further away from the resonance, exactly what one should expect for a size-dependent Yarkovsky effect and a size-independent chaotic diffusion.

In this framework, if we want to correctly evaluate the number of asteroids supplied by the Yarkovsky effect to the resonance, we need to measure the flux of asteroids into the diffusive region, and not that into the resonance itself. For this reason, we shift the boundaries of the $3 / 1$ resonance 0.015 AU away from the nominal resonance borders (dashed lines in the left panel of Fig. 3). In doing this we implicitely assume that all the bodies entering the diffusive neighborhood will eventually fall into the resonance, neglecting the possibility of their collisional disruption in the diffusion zone. This should not be a severe simplification, given that the typical collisional lifetimes for 1- and 10-km bodies are of order 350 and $1200 \mathrm{Myr}$, respectively (see formula (9) in Section 5).

For the $\nu_{6}$ resonance, the numerical integrations by Morbidelli and Gladman (1998) and Nesvorný et al. (2002) indicate that chaotic diffusion is a relevant phenomenon in the $0.05-$ to $0.10-\mathrm{AU}$ neighborhood. An analysis similar to that shown in the left panel of Fig. 4 shows that the density of asteroids grows until $0.08-0.09 \mathrm{AU}$ from the resonance. To measure the flux of Yarkovsky-driven multikilometer asteroids we therefore drift the $\nu_{6}$ resonance boundary outward by $0.09 \mathrm{AU}$, as indicated by the dashed curve in the right panel of Fig. 3. In the following we will refer to these shifted boundaries simply as "resonant boundaries." In Section 6 we will discuss how the specific choice of the boundaries influences our final results.

\section{Modeling the Yarkovsky and the YORP effects}

The major orbital perturbation caused by the Yarkovsky effect is a secular change of the semimajor axis (e.g., Bottke et al., 2003). For asteroids with low surface thermal conductivity $\left(\approx 10^{-3} \mathrm{~W} / \mathrm{m} / \mathrm{K}\right)$, a reasonable assumption for kilometer size and larger bodies, likely covered with a regolith layer (e.g., Müller and Lagerros, 1998), the diurnal variant of the effect dominates. The latter can both secularly decrease and increase the semimajor axis, depending on their obliquity being larger or smaller than $90^{\circ}$. The semimajor axis drift speed is computed with the formula

$$
\frac{d a}{d t}=k(R, K, \ldots) \cos \epsilon,
$$

where $\epsilon$ is the obliquity of the body's spin axis and $k$ is function of the radius $R$, thermal conductivity $K$ of the surface, and several other thermal parameters. The exact analytic form of $k$ may be found in Vokrouhlický (1998, 1999), while Fig. 5 shows its value in a graphic form for a 1 -km-diameter body with $2.5 \mathrm{~g} / \mathrm{cm}^{3}$ bulk density, as a function of $K$. The vertical dashed lines bound the range of values for $K$ that are consistent with IR observations (Müller and Lagerros, 1998), and the vertical dotted line indicates the nominal value of $K$ that we will use in the simulations, which gives, for an obliquity of $0^{\circ}$ or $180^{\circ}$, a semimajor axis drift rate of $\pm 2.7 \times 10^{-4} \mathrm{AU} / \mathrm{Myr}$. This drift rate scales almost inversely with the characteristic size $(k \propto 1 / R)$ and 


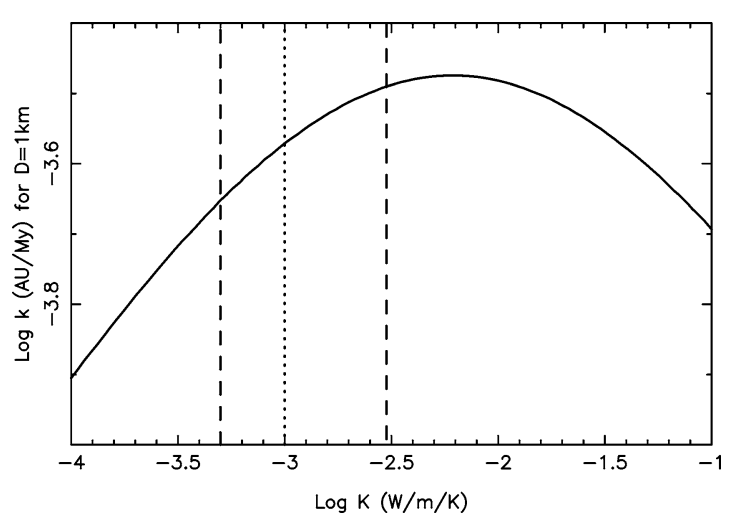

Fig. 5. The maximum drift rate $k$ as a function of the thermal conductivity $K$, for a $1-\mathrm{km}$-diameter body with $2.5 \mathrm{~g} / \mathrm{cm}^{3}$ bulk density. The range of expected values of $K$ for main belt asteroids is bounded by the dashed vertical lines, while the dotted line shows the nominal value assumed in our simulations.

becomes thus practically unimportant for objects larger than $\simeq 20 \mathrm{~km}$ in diameter.

The values of $k$ shown in Fig. 5 have been computed for spherical bodies. The corresponding values averaged over a large sample of irregularly shaped test bodies using the Gaussian random spheres method (Muinonen and Lagerros, 1998) and using a nonlinear theory of the heat diffusion are estimated to be $\sim 25 \%$ smaller (Vokrouhlický and Čapek, 2003 , to be submitted). Because $25 \%$ is approximately the uncertainty of the drift rate due to the lack of precise knowledge of the thermal conductivity, we neglect the nonsphericity effects.

The Yarkovsky effect depends on a number of physical and geometric parameters, and we must make a specific choice of these parameters in our simulations. We noted already that in the case of kilometer-scale asteroids a low value of the surface conductivity is justified and we shall use it throughout our simulations. We also mentioned the obliquity dependence; except for simulations incorporating the YORP effect described in the following, we assume random orientation of the spin axes in space, implying a uniform distribution in $\cos \epsilon ; \epsilon$ is assumed time-independent until a sufficiently energetic, but still nondisruptive collision, reorients the spin axis direction. Reorienting collisions have been introduced by Farinella et al. (1998), who defined them as collisions exchanging with the target an amount of angular momentum equal, on average, to the target's rotational angular momentum. The charateristic time scale of such events can be given as

$$
\tau_{\text {reor }} \simeq B R^{\beta}
$$

where $R$ is the radius of the target; if the latter is given in meters, the projectile population is assumed to follow the Dohnanyi (1969) equilibrium distribution of sizes, and the rotation frequency scales as $1 / R$, Farinella et al. argued that $B \simeq 15$ Myr and $\beta=0.5$ (see also Farinella and Vokrouhlický, 1999). However, given the large uncertainties in these quantities, we consider them as free parameters and will explore the sentivity of the results on their values. After a reorienting collision, the spin axis obliquity is randomly reset, according to a uniform distribution in $\cos \epsilon$.

The diurnal variant of the Yarkovsky effect depends also on the rotation rate $\omega$, though only weakly (as $\sqrt{\omega}$; Vokrouhlický, 1998, 1999; Bottke et al., 2003). The observed distribution of the rotation rates of small asteroids in the 1to $10-\mathrm{km}$ range indicates only a very weak size dependence (Pravec and Harris, 2000). We thus assume that asteroids in our simulation have nearly size-independent rotation rates; we assume values in the range 3 to $15 \mathrm{~h}$, to prevent excess due to very fast or slow rotators, with a broad Gaussian distribution in $\omega$ peaked at $\simeq 6 \mathrm{~h}$. The true distribution of small-asteroid $\omega$ deviates from Gaussian (Pravec and Harris, 2000); nevertheless, given other uncertainties in our study, and given the weak dependence of the Yarkovsky effect on the rotation rate in the considered range, we stay with the assumption of the Gaussian distribution. We nevertheless verified the robustness of our conclusions by initially putting a flat probability distribution of the rotation rates for the asteroids, without noticing any difference from the "nominal results."

Rubincam (2000), followed by Vokrouhlický and Čapek (2002), argued that the thermal recoil force which is the basis of the Yarkovsky orbital effect also results in a longterm secular change of the asteroids rotation state, provided the body is of irregular shape. Rubincam suggested calling this effect YORP as an acronym to Yarkovsky-O'KeefeRadzievski-Paddack, the last three contributing to the development of a related model for the radiation pressure in the 1960s. YORP secularly modifies obliquity and rotation rate, both relevant quantities for determining the strength of the Yarkovsky perturbation of the semimajor axis. The "tricky" circumstance of the YORP effect is that it sensitively depends on the shape; in fact it vanishes for both spherical and spheroidal bodies. To evaluate the averge importance of the YORP effect over a large sample of bodies, Vokrouhlický and Čapek (2002) determined its mean value over 500 synthetic bodies, generated using the Gaussian spheres method introduced by Muinonen and Lagerros (1998), which reasonably well reproduces the shapes of kilometer scaled asteroids. This result, derived with a simplified assumption of zero surface thermal condutivity, has been recently generalized by Čapek and Vokrouhlický (2003, to be submitted) for bodies with finite surface thermal conductivity. In summary, the YORP effect can be described as follows: Given arbitrary initial conditions the obliquity is driven toward an asymptotic value of $0^{\circ}$ or $180^{\circ}$; during this process the rotation rate is changed; by the time the obliquity has reached a nearly asymptotic value, the rotation rate has decelerated or accelerated (with deceleration more likely, occurring in some $60 \%$ of the cases). Obviously, this evolution assumes that only YORP is acting; Rubincam (2000) conjectured that during the asymptotic state, the YORP evolution would be interrupted by 


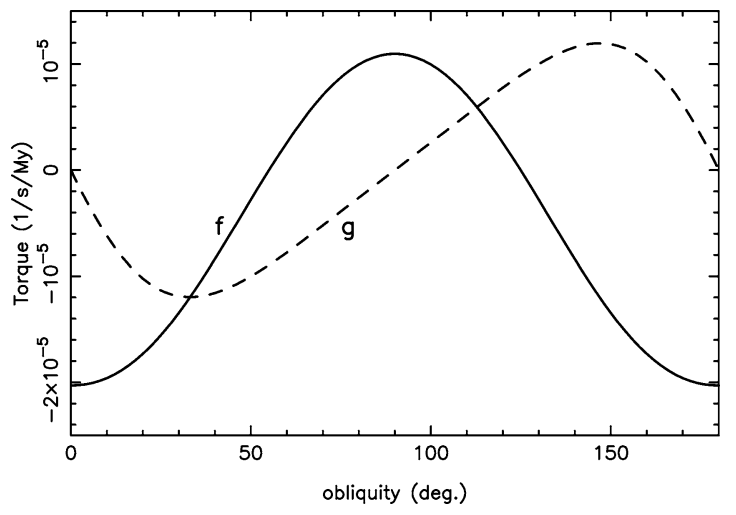

Fig. 6. The functions $f$ and $g$ of Eqs. (7) and (8) for the $\sim 60 \%$ of the bodies that eventually decelerate their spin rate. For the other $40 \%$ that eventualy accelerate, the function $f$ is changed of sign. The ordinate units are $1 / \mathrm{s} / \mathrm{Myr}$, for convenience.

rotational fission (in the case of accelerating rotation) or by collisional reorientation (in the case of decelerating rotation). In the latter case, the evolution restarts from the new state and then follows a new evolution track (a scenario termed "YORP cycle" by Rubincam (2000)).

To see the influence of the YORP evolution in our model for the origin of NEAs, we implement these concepts in the following form. The evolution of the obliquity $\epsilon$ and the rotation rate $\omega$ is given by

$$
\begin{aligned}
& \frac{d \omega}{d t}=f(\epsilon), \\
& \frac{d \epsilon}{d t}=\frac{g(\epsilon)}{\omega},
\end{aligned}
$$

where the functions $f$ and $g$ are the time averages of the radiation torque, projected onto the direction of the spin axis and a direction perpendicular to it (Rubincam, 2000, Vokrouhlický and Čapek, 2002). Obviously, they depend on the particular shape of the body, but here we use their average values over the large sample of bodies generated with the Gaussian sphere method, computed by Vokrouhlický and Čapek (2002); see Fig. 6. We note that these results still assume zero surface thermal conductivity; also, no attempt to include further effects, such as temporary captures in secular spin-orbit resonances are made in this study.

Quantitatively, we are mainly concerned with two relevant time scales. The first one is that of the evolution that drives the obliquity toward its asymptotic state, which enters in a direct way in the computation of the Yarkovsky effect. The other time scale concerns the asymptotic spindown or spin-up of the rotation rate, which enters in a more indirect way into the simulation. In the case of spin-down, the spin momentum is decreased, so that the projectiles that can reorient the body are smaller than in the YORP-less case. This fact, given the size distribution of the asteroid population, results in a reduction of the characteristic reorientation time scale. In the case of spin-up, rotational fission eventualy occurs; in our model we assume that this is the case whenever the rotation period decreases below $2 \mathrm{~h}$. Vokrouhlický and Čapek (2002) showed that these time scales are comparable and amount to $\approx 10-50 \mathrm{Myr}$ for a kilometer-size asteroid at $\simeq 2.5 \mathrm{AU}$. They also noted that this value does not vary significantly in the asteroid belt, since the smaller solar radiation flux in its outer part is nearly compensated by systematically smaller bulk density of asteroids (given taxonomic differentiation). Therefore, the most important dependence of the YORP characteristic time scale is on size, scaling with radius $R$ as $f, g \propto 1 / R^{2}$. This rapid regression makes the YORP effect unimportant for bodies with $R \geq 5 \mathrm{~km}$.

\section{Recipe for the simulation of a steady-state process}

As anticipated in the introduction, our simulation is done with a combination of numerical integration of the Yarkovsky equations and Monte Carlo techniques, as in Vokrouhlický and Farinella (2000). We neglect all planetary perturbations and let the body drift in semimajor axis under the Yarkovsky effect, at constant eccentricity and inclination.

If we took into account the sole Yarkovsky effect, because of the $\sim 1 / D$ dependence of the drift rate, the size distribution of the bodies transported into the resonance would be steeper than the size distribution of main belt bodies by a factor $1 / D$. This would give a $H$ distribution of type (1) with $\gamma=0.2+\gamma^{\prime}$, with $\gamma^{\prime}$ being the corresponding coefficient in the exponent of the $H$ distribution for the main belt. Assuming $\gamma^{\prime}=0.25$ for $15.5<H<18$ as in Section 2 , this would give $\gamma=0.45$. This is considerably larger than the slope observed in the NEA population (0.35-0.39; Rabinowitz et al., 2000; Bottke et al., 2000b, Stuart, 2001). However, the reality is much more complex. The bodies undergo collisional reorientations of their spin axes (which reset their drift speeds) and are collisionally disrupted on their way to the resonance. Because both phenomena occur more frequently for small bodies than for large ones, it is reasonable to expect that they reduce the difference between $\gamma^{\prime}$ and $\gamma$. But a simulation incorporating collisional reorientations and disruptions would inevitably produce a flux into the resonances that decays with time. (More precisely, reorientations alone would give a flux decaying as $1 / \sqrt{t}$, while collisional disruptions would give a flux decaying exponentially in time.) Hence, to have a realistic estimate of the current rate at which the resonances are resupplied, we need to take into account that the asteroids are also collisionally regenerated by the breakup of bigger bodies. This regeneration rate on average should equilibrate the loss rates due to collisional disruption and escapes into the NEA space, maintaining the belt in an approximate steady state. (Recall that the existence of a steady state is a usual assumption, driven by the evidence that the NEA population 
has been roughly constant over the last 3.5 Gyr (Grieve and Shoemaker, 1994; Neukum and Ivanov, 1994).)

Ideally, we should develop a code that at the same time simulates the Yarkovsky-driven drift of the asteroids in orbital space, their spin-axis reorientations, and the collisional cascade ongoing in the asteroid belt. Because our goal is to provide a first evaluation of the role played by the Yarkovsky effect for the origin of NEAs, we believe that this approach would be too ambitious. Rather, we have preferred to adopt a simpler strategy: We have designed a model that asymptotically evolves toward a steady state which, for the point of view of total number of asteroids, orbital, and absolute magnitude distribution, is equivalent to the main belt model generated in Section 2. Our recipe is as follows.

We start from the main belt model detailed in Section 2. At each time step $d t$ of the temporal evolution (usually equal to $0.5 \mathrm{Myr}$ ) we perform the following operations:

(i) Assuming that for a body with radius $R$ the average time scale of collisional disruption is

$$
\tau_{\text {disr }}=A R^{\alpha}
$$

(the values of $\mathrm{A}$ and $\alpha$ depending on the simulation; see Section 6), for each asteroid in the simulation we generate a random number between 0 and 1 ; if the latter is smaller than $1-\exp \left[-d t / \tau_{\text {disr }}\right]$ we assume that a collisional disruption occurs. Considering that in a steady state for each collisional disruption there should be a collisional regeneration, we reintroduce the body in the simulation, keeping invariant its absolute magnitude $H$, and attributing new $a, e$, $i$, randomly chosen from the set of values characterizing the initial population (Section 2). The diameter is computed using the characteristic albedo for the new semimajor axis zone. A new obliquity $\epsilon$ is randomly chosen from a uniform distribution in $\cos \epsilon$, and a new rotation rate $\omega$ is chosen from the distribution discussed in Section 4.

(ii) For the asteroids which have not been disrupted during the time step, we compute the new semimajor axis according to the drift rate given in (5). If a body crosses a resonant boundary during the time step it is recorded as a new resonant body and is eliminated from the simulation. However, in a steady state the dynamical loss of bodies must be compensated by the collisional generation of new objects. The latter occurs on a fixed characteristic time scale (see Eq. (9)), independent of the dynamical fate of the bodies. In practice, we implement this situation by putting the resonant bodies into a "resonant buffer," where they wait until the algorithm described in (i) dictates their reintroduction in the system.

(iii) If the YORP effect is taken into account (which occurs only in some of the simulations; see Section 6) we compute for each body the new obliquity and rotation period using (7) and (8). The new values are used for the computation of the semimajor axis drift rates during the next time step. If the orbital period has decreased below $2 \mathrm{~h}$, the body is assumed to undergo rotational fission. In this case the body is reintroduced in the simulation with new orbital elements and spin parameters, as for the bodies undergoing collisional disruption (see point (i)).

(iv) Assuming a characteristic size-dependent reorientation time scale $\tau_{\text {reor }}$ (see Eq. (6)), for each asteroid we generate a random number between 0 and 1; if the latter is smaller than $1-\exp \left[-d t / \tau_{\text {reor }}\right]$ we assume that a reorientation occurs and we attribute to the asteroid a new obliquity $\epsilon$ and a new rotation rate $\omega$.

It is evident that with this algorithm the system is forced to evolve toward a steady state, because each body that leaves the system is reintroduced with parameters (initial orbital elements, obliquity, and rotation rate) that are randomly chosen according to the distributions that characterize the initial population. If there were no dynamical exit routes (the resonance regions), the steady-state population would be statistically equivalent to the starting population. With the exit routes active, the steady-state population may differ from the initial one in the vicinity of the resonant boundaries. The width of the region where the difference can be significant depends on the reorientation and disruption time scales. If the latter are large enough, it may be a significant portion of the entire belt. In these cases, we redo the simulation changing the $H$ distribution of the initial population (but preserving the orbital distribution), until the final steady-state population has the same total number of asteroids and $H$ distribution of our main belt population model (see Section 2). Because the simulations show simple relations between the initial and the final distributions, the desired result is usually attained in a couple of iterations. A visual inspection of the steady-state orbital distribution also shows a quite nice similarity with the orbital distribution of our main belt model. In particular, all major asteroid families can be easily recognized.

The advantage of our toy model is that it is fast and effective. Moreover, we do not need to bother about the usual tricky parameters of the collisional evolution codes, such as those related to impact strength, ejection velocity fields, and size distribution of fresh collisional debries, which are all poorly constrained. The limitations are obvious, though. While a proper simulation of the evolution of the belt may provide statistical information on the evolution of the NEA generation rate over the age of the Solar System (both for what concerns a possible secular trend and random oscillations), our toy model can just simulate the current steady-state situation. The transition phases toward the steady state in our simulation should not be interpreted as a representation of the past history of the belt and of the NEA population. Only the final steadystate situation is relevant for our purposes.

\section{The flux of multikilometer asteroids into the $3 / 1$ and $\nu_{6}$ resonance, and the NEA size distribution}

We now come to analyze the results of our simulations and discuss their dependence on the main free parameters of the model. 

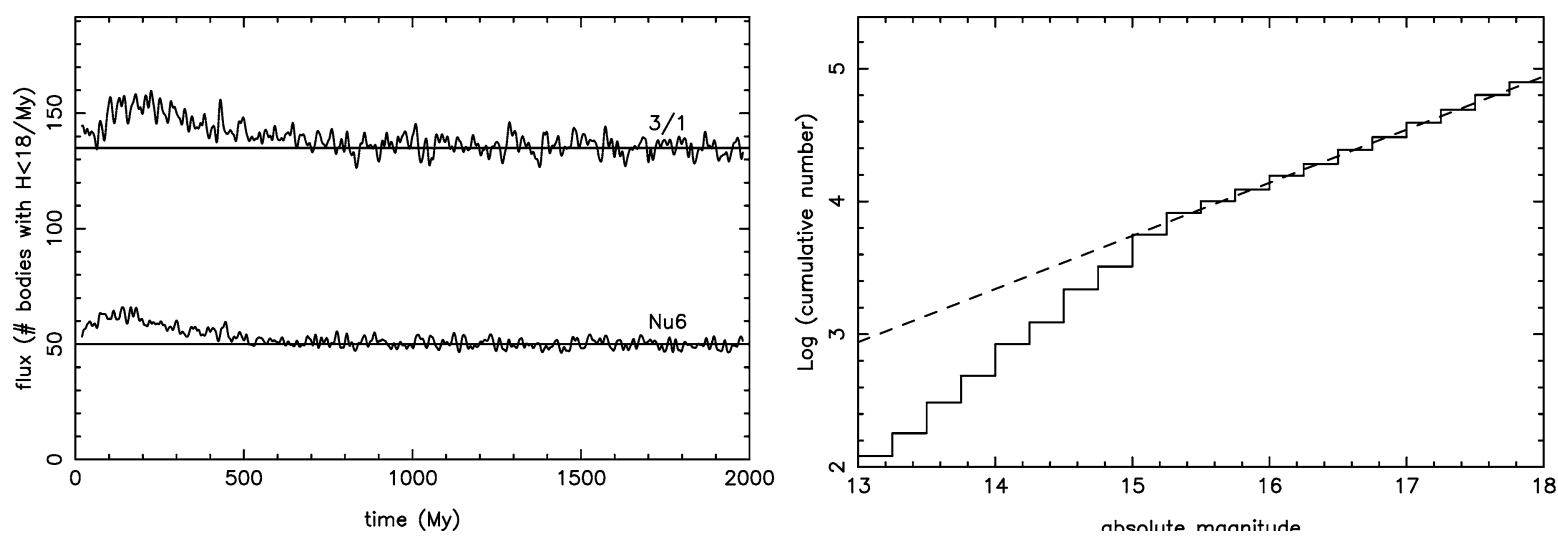

Fig. 7. (Left) The flux of bodies with $H<18$ into the $3 / 1$ and $\nu_{6}$ resonances in the course of the 2-Gyr simulation. A steady-state situation is rapidly reached. The horizontal lines show the average steady-state fluxes of 135 and 50 bodies per million years, respectively. (Right) The cumulative $H$ distribution of the bodies that enter the $3 / 1$ or the $\nu_{6}$ resonance in the last $0.5 \mathrm{Gyr}$ of simulation. The dashed line has slope $\gamma=0.40$ and fits the distribution in the $15.5<H$ $<18$ range.

As a "nominal simulation" we consider the case with disruption and reorientation time scales given by the relationships (9) and (6), with coefficients $A=16.8 \mathrm{Myr}, \alpha=$ $1 / 2, B=15 \mathrm{Myr}$, and $\beta=1 / 2$ and the radius $R$ measured in meters. These formulas have already been used in Farinella and Vokrouhlický (1999). The YORP effect is not taken into account. The solid and dashed curves in the left panel of Fig. 7 show the flux of $H<18$ bodies into respectively the $3 / 1$ and the $\nu_{6}$ resonance as a function of time, in the course of our 2-Gyr integration. After some transient phase in the first part of the simulation, these fluxes oscillate around a fairly constant value, which means that our simulation has effectively converged toward a steady-state situation. The resulting fluxes are 135 bodies with $H<18$ per Myr in 3/1 and 50 in $\nu_{6}$. These fluxes compare very well with those deduced by Bottke et al. (2002) on the basis of their NEA model: respectively to $100 \pm 50$ and $55 \pm 18$. The cumulative magnitude distribution of the bodies that entered the $3 / 1$ or the $\nu_{6}$ resonance during the last $0.5 \mathrm{Gyr}$ of simulation (after that the steady state has been reached) is illustrated in the right panel of Fig. 7. The exponent coefficient $\gamma$ of the exponential distribution (1) is 0.40 , for 15.5 $<\mathrm{H}<18$. This value is steeper than the exponent coefficient of the NEA population according to Rabinowitz et al. (2000) and Bottke et al (2000b) $(\gamma=0.35)$, but well comparable to that estimated by Stuart (2001), i.e., $\gamma=0.39$.
We have done a series of 11 other simulations, changing the $A$ and $B$ coefficients by the factors reported in Table 1 . The results for the fluxes and for the exponent of the $H$ distribution, computed over the last 0.5 Gyr of simulation, are reported in Table 1. We remark that, while the exponent of the magnitude distribution is essentially the same in all cases, the fluxes significantly increase when the reorientation time scale is increased. The disruption time scale has a more moderate importance. If the reorientation time scale is short, then the highest fluxes are obtained with a comparatively short disruption time scale. If the reorientation time scale is the nominal one or larger, then the largest fluxes are obtained with the longest disruption lifetime. When the reorientation time scale is short, the motion of the bodies through the belt resambles a random walk. Consequently, as Fig. 8 shows, the fluxes into the resonances decay as $1 / \sqrt{t}$, before a steady-state situation is achieved. As said in the previous section, our simulations do not have the ambition to reproduce the evolution of the NEA flux over the age of the Solar System; the decay phase should just be considered as a transient phase of the simulation, and only the final steady-state rates should be considered as relevant.

To investigate the sensitivity of the results to the shift imposed in Section 3 to the resonant boundaries, we have also performed a nominal simulation but assuming that the $3 / 1$ boundaries are shifted only by $0.005 \mathrm{AU}$ and the $\nu_{6}$

Table 1

Summary of results concerning simulations which assume $\tau_{\text {disr }}$ and $\tau_{\text {reor }}$ as in (9) and (6), but with coefficients $A$ and $B$ scaled with respect to the "nominal values" $(A=16.8 \mathrm{Myr}, B=15 \mathrm{Myr})$ by the factors reported in the first column and the first line below

\begin{tabular}{|c|c|c|c|c|c|c|c|c|c|c|c|c|}
\hline \multirow{2}{*}{$\begin{array}{l}\mathrm{B} \rightarrow \\
\downarrow \mathrm{A}\end{array}$} & \multicolumn{3}{|l|}{0.1} & \multicolumn{3}{|l|}{0.3} & \multicolumn{3}{|l|}{1} & \multicolumn{3}{|l|}{3} \\
\hline & $F_{3 / 1}$ & $F_{\nu 6}$ & $\gamma$ & $F_{3 / 1}$ & $F_{\nu 6}$ & $\gamma$ & $F_{3 / 1}$ & $F_{\nu 6}$ & $\gamma$ & $F_{3 / 1}$ & $F_{\nu 6}$ & $\gamma$ \\
\hline 0.3 & 89 & 36 & 0.40 & 112 & 45 & 0.40 & 129 & 51 & 0.40 & 137 & 54 & 0.40 \\
\hline 1 & 72 & 29 & 0.40 & 104 & 41 & 0.40 & 135 & 50 & 0.40 & 149 & 54 & 0.40 \\
\hline 3 & 57 & 23 & 0.40 & 91 & 33 & 0.40 & 136 & 47 & 0.39 & 161 & 52 & 0.38 \\
\hline
\end{tabular}

Note. For each simulation, the steady-state flux into the $3 / 1$ resonance $\left(F_{3 / 1}\right)$ and $\nu_{6}$ resonance $\left(F_{\nu 6}\right)$ are reported in number of bodies with $H<18$ per million years. The exponent coefficient $\gamma$ of the $H$ distribution (1) of the bodies captured into resonance over the last 0.5 Gyr of simulation is also given. 

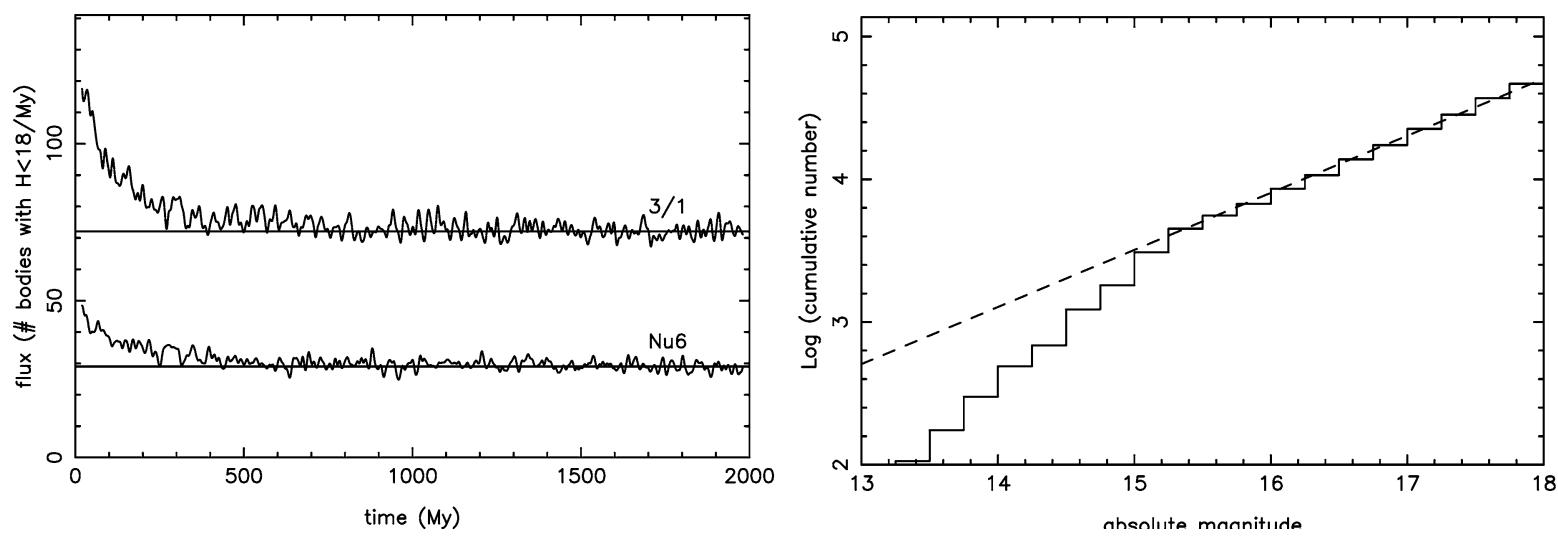

Fig. 8. The same as in Fig. 7, but for the case where the reorientation time scale has been decreased by a factor of 10 .

boundary by $0.03 \mathrm{AU}$. The resulting fluxes into the resonances are moderately reduced, giving 129 bodies in $3 / 1$ and 36 in $\nu_{6}$. This was expected because of the reduced population in the resonant neighborhoods. No substantial change in the $H$ distribution of the resonant objects is observed.

In a series of seven other simulations we explore the dependence of the results on $\beta$, the exponent of $R$ that appears in the formula (6). The nominal value $(\beta=1 / 2)$ is obtained (Farinella et al., 1998) assuming that the rotation speed $\omega$ is-on average-inversely proportional to $R$ and that the overall cumulative size distribution in the main belt is $N(>R) \propto R^{-2.5}$ (Dohnanyi, 1969). According to modern data (Pravec and Harris, 2000), the rotation speed seems to be roughly independent on $R$, and it is not clear what the overall size distribution is in the main belt, averaged over a large size range. Hence we think that the value of $\beta$ is poorly constrained. Table 2 lists the values of $\beta$ that we have considered and the resulting exponent $\gamma$ of the magnitude distribution of the bodies captured into one of the considered resonances. The numerical coefficient $B$ in (6) has been chosen in all cases so that the reorientation time scale for bodies with $R=500 \mathrm{~m}$ is the same as in our nominal case. The assumed disruption time scale is also our nominal one. Because of these choices, the resulting fluxes in the $3 / 1$ and $\nu_{6}$ resonances are essentially identical to those of the nominal simulation (see the left panel of Fig. 7). Interestingly, Table 2 shows that the larger $\beta$ is, the smaller $\gamma$ is. This is because the small bodies undergo more frequent reorientations compared to the larger ones, which preferentially inhibits their transport toward the resonances. For $\beta \geq$ 0.75 the resulting $H$ distribution is compatible with the slope of the NEA population according to Stuart (2001) but still substantially steeper than the $H$ distributions found by Rabinowitz et al (2000) and Bottke et al. (2000b).
We have also done a similar exploration concerning $\alpha$, the exponent of $R$ appearing in the formula for the disruption time scale $\tau_{\text {disr }}$. But in this case we have not found any significant variation on $\gamma$.

We move now to consider how the situation changes if the YORP effect is also taken into account. In this new simulation we use the nominal disruption time scale and an $\omega$-dependent reorientation time scale given by

$$
T_{\text {reor }}=15\left(\frac{1}{500} \frac{\omega}{\omega_{0}}\right)^{5 / 6} R^{4 / 3}
$$

with $\omega_{0}=2 \pi /(5 \mathrm{~h})$. This formula is due to Farinella et al. (1998) and reduces to our nominal reorientation law (Eq. (6) with $B=15 \mathrm{Myr}$ and $\beta=1 / 2$ ) if one assumes that $\omega$ scales as $1 / \mathrm{R}$ and is equal to $2 \pi /(5 \mathrm{~h}$ ) for a $R=500 \mathrm{~m}$ body. The $f$ and $g$ functions entering the YORP equations (7) and (8) are those illustrated in Fig. 6. The results of this simulation are illustrated in Fig. 9. As for the case of fast reorientations without the YORP effect (Fig. 8), at the beginning of the simulation the fluxes into the resonances drop as $1 / \sqrt{t}$, until a steady state is reached. The steady-state fluxes are equal to 109 bodies with $H<18$ per million years for the 3/1 resonance and 39 for the $\nu_{6}$ resonance, very similar to those determined by Bottke et al. (2002). The exponent coefficient of the $H$ distribution (1) of the bodies captured into resonance during the last $0.5 \mathrm{Gyr}$ of simulation is $\gamma=0.335$. Of all the simulations analyzed in this paper, this is the one giving the shallowest NEA magnitude distribution, with exponent smaller than 0.35 (the value determined by Rabinowitz et al. (2000) and Bottke et al. (2000b) from the observed NEA population). The reason for this shallow distribution is that, as explained in Section 4, the YORP

Table 2

Summary of results concerning simulations which assume different values for the exponent $\beta$ in (6)

\begin{tabular}{|c|c|c|c|c|c|c|c|c|c|}
\hline$\beta$ & -0.5 & -0.25 & 0 & 0.25 & 0.5 & 0.75 & 1 & 1.25 & 1.5 \\
\hline$\gamma$ & 0.44 & 0.43 & 0.42 & 0.41 & 0.40 & 0.39 & 0.39 & 0.38 & 0.37 \\
\hline
\end{tabular}

Note. The assumed disruption time scale is our nominal one. The second line reports the value of $\gamma$ in the resulting NEA magnitude distribution (1). 

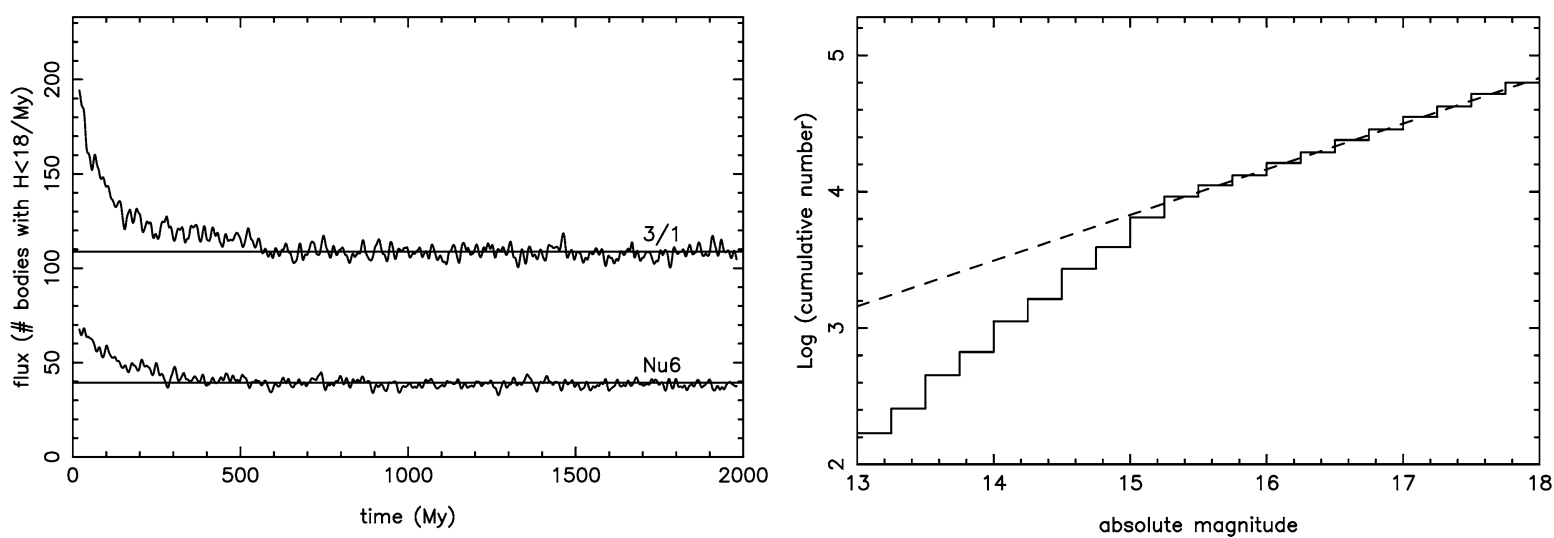

Fig. 9. The same as in Fig. 7, but for the case where the YORP effect has been taken into account with the $f$ and $g$ functions illustrated in Fig. 6. The dashed line in the right panel has slope $\gamma=0.335$.

effect accelerates or decelerates the spin rates, causing the disruption or the reorientation of the bodies. This happens on a time scale proportional to $R^{2}$. Due to this fast progression of the YORP evolution time scale with $R$, the Yarkovsky drift of small bodies becomes inhibited relative to the larger bodies. This directly results in a shallower magnitude distribution of the resonant captured bodies. In summary, the inclusion of the YORP effect goes in the same direction of increasing the exponents $\alpha$ and $\beta$ in the disruption and reorientation formulas (9) and (6), which-as shown in Table 2-reduces $\gamma$.

Because large uncertainties still exist in the modeling of the YORP effect, we have perfomed six additional simulations, in which we decrease the $f$ and $g$ functions with respect to those shown in Fig. 6 by the factors reported in Table 3. The corresponding fluxes into $3 / 1$ and $\nu_{6}$ resonances and the slope of the cumulative $H$ distribution of the resonant objects are reported in the same table. We stress that, despite the mentioned uncertainties, a realistic range for these factors should be $0.2-1$, unless our current understanding of the YORP effect is largely mistaken. The

Table 3

Summary of the results of the simulations that account for the YORP effect

\begin{tabular}{llll}
\hline $\mathrm{c}^{\mathrm{a}}$ & $F_{3 / 1}{ }^{\mathrm{b}}$ & $F_{\nu 6}{ }^{\mathrm{b}}$ & $\gamma^{\mathrm{c}}$ \\
\hline 1 & 109 & 39 & 0.335 \\
0.5 & 128 & 46 & 0.345 \\
0.2 & 163 & 60 & 0.360 \\
0.1 & 179 & 63 & 0.380 \\
0.02 & 166 & 59 & 0.395 \\
0.0005 & 147 & 54 & 0.395
\end{tabular}

\footnotetext{
${ }^{\text {a }}$ The factor by which the $f$ and $g$ functions of Fig. 6 are multiplied.

${ }^{\mathrm{b}} F_{3 / 1}$ and $F_{\nu 6}$ denote the corresponding fluxes (number of bodies with $H<18$ per million year) into the $3 / 1$ and $\nu_{6}$ resonances.

${ }^{\mathrm{c}}$ The exponent coefficient of the $H$ distribution (1) in the 15.5-18 range of the bodies captured into resonances during the last $0.5 \mathrm{Gyr}$ of simulation.
}

smaller reduction factors listed in Table 3 have been used for understanding how the results approach those of the "nominal" simulation of Fig. 7, which did not account for YORP.

As one sees from Table 3, decreasing the magnitude of the YORP effect increases the slope of the NEA distribution. In particular if the $f$ and $g$ functions are divided by a factor 2, the agreement with the NEA slope of Rabinowitz et al. and Bottke et al. is excellent. If they are divided by a factor 50 or more, the resulting $\gamma$ is essentially the same as in the "nominal" simulation. This was expected from the aforementioned considerations, because decreasing the YORP effect effectively increases the reorientation and disruption time scales of the smallest bodies, relative to the big bodies.

The changes to the resonant fluxes are more intriguing. As the YORP effect is decreased, the fluxes first increase and then decrease. The initial increase of the fluxes is correlated with the steepening of the magnitude distribution and is the consequence of the larger mobility of the small bodies determined by the longer reorientation and disruption time scales. We notice that if the $f$ and $g$ functions are divided by a factor 5 to 50 the fluxes even exceed those of Fig. 7. This is because the YORP effect breaks the uniform distribution of obliquities, thus favoring the existence of fast drifting bodies. If the YORP effect is further decreased, its role eventually becomes negligible and the fluxes decrease back to the nominal values of Fig. 7 .

Finally, in the case of our "nominal" YORP simulation (c $=1$ in Table 3), we have tested the dependence of the results on the semimajor axis drift rate (the function $k$ in Eq. (5)). The fluxes into the resonances scale almost linearly with the drift rate, despite the nonobvious feedback introduced by the steady-state model. Therefore, the $25 \%$ uncertainty of the drift rate (due to our lack of knowledge of the asteroidal thermal conductivity) linearly reflects onto all the results reported herein. 


\section{Conclusions and discussion}

In this paper we have investigated the role of the Yarkovsky effect in the origin of near-Earth asteroids. We have designed an original simulation scheme that allows us to compute the "current steady-state" fluxes of $H<18$ asteroids (roughly larger than $1 \mathrm{~km}$ in size) into the most important NEA source resonances. By "current steady-state" we mean a steady-state situation in which the main belt has its current properties. In particular, we have not tried to investigate how the asteroid flux changes when an asteroid family forms nor have we tried to reconstruct the evolution of the NEA population over the age of the Solar System.

The simulations depend on a number of poorly constrained parameters, such as the collisional disruption and reorientation time scales, their size dependence, and the average magnitude of the Yarkovsky and YORP effects. The latter is a variant of the Yarkovsky effect, which acts on the spin axis obliquity and rotation frequency of the bodies. Therefore, we have explored the sensitivity of the results on the values of these parameters. The results also depend on the assumed properties of the current main belt population, in particular the total number of bodies with $H<18$ and their magnitude distribution. In the reported simulations we have used the main belt model described in Section 2 but the dependence of the results on the main belt parameters is quite straightforward and will be discussed in the following.

With the exception of the cases with a very fast reorientation time scale (a tenth of the nominal values), our estimated fluxes into the $3 / 1$ and $\nu_{6}$ resonances are quite large. About 100-160 bodies with $H<18$ should enter the 3/1 resonance every million years while $40-60$ should enter the $\nu_{6}$ secular resonance. These rates are similar to those independently derived by Bottke et al. (2002) on the basis of their NEA population model $(100 \pm 50$ and $55 \pm 18$ respectively). However, in our simulations, the ratio between the fluxes into $3 / 1$ and $\nu_{6}$ is in the range 2.5 to 3.0, while in Bottke et al. it is $1.8 \pm 0.75$. But it should be taken into account that Bottke et al. had difficulty in distinguishing NEAs coming from the $v_{6}$ resonance from those coming from the Mars-crossing population, so that it might be possible that the former contribution has been somewhat overestimated.

The Yarkovsky-driven fluxes reported here have been obtained assuming that there are 563,000 asteroids with $H$ $<18$ in the belt with a $<2.8 \mathrm{AU}$, implying some $1,300,000$ bodies in the entire belt. A population of about 1,000,000 $\mathrm{H}$ $<18$ bodies would still give fluxes consistent with Bottke et al. rates. However, had we assumed a main belt population containing only 520,000 $H<18$ bodies, as claimed by Ivezić et al. (2001), the fluxes into the resonances would have been $40-65$ and $16-24 H<18$ bodies per million years respectively, far too small with respect to the Bottke et al. values. This seems to support the suspicion that there might be a problem in the conversion between the number of bodies detected by the SDSS survey and the total number of bodies in the belt. We remark however that if the estimate by Ivezić et al. is correct, then there is a serious problem in the absolute magnitudes reported in the asteroid catalogs (see Section 2 for a discussion). In this case it is plausible that a similar problem also exists in the NEA catalog, so that the real number of discovered NEAs with $H<18$ would be considerably smaller than the one currently claimed. This would have the consequence of reducing the total number of $H<18$ NEAs in the models of Rabinowitz et al. (2000), Bottke et al. (2000b), and Stuart (2001). Therefore, the estimates of the resonant fluxes in Bottke et al. (2002) would be comparatively reduced as well. It is unclear, however, if the new fluxes would decrease as low as $\sim 50$ and $\sim 20$ bodies per million years in the $3 / 1$ and $\nu_{6}$ resonances respectively, to be consistent with our model.

To conclude whether the Yarkovsky effect can produce the observed NEA population, a second aspect to be considered is the $H$ distribution of the bodies captured into resonance. In the simulations that do not account for the YORP effect and assume that the reorientation time scale is proportional to the square root of the size of the bodies, the slope coefficient $\gamma$ of the $H$ distribution (1) is $0.39-0.40$ for $15.5<H<18$. This is compatible with the slope of the real NEA population (0.39) according to Stuart (2001) but is steeper than that (0.35) derived by Rabinowitz et al. (2000) and Bottke et al. (2000b). If the dependence on size of the reorientation time scale is steepened, the resulting value for $\gamma$ is decreased (Table 2) and may approach 0.35. Finally, if the YORP effect is taken into account, with nominal or slightly reduced strength (up to a factor 2), then $\gamma \lesssim 0.35$. We consider that the simulations accounting for the YORP effect are superior, because they account for an additional real dynamical phenomenon. We stress that taking into account the Yarkovsky effect but not the YORP effect is, strictly speaking, inconsistent.

It should be qualified that the values of $\gamma$ discussed here have been obtained assuming that the $H$ distribution of the main belt population in the $15.5<H<18$ range has slope $\gamma=0.25$. This is the slope determined by Jedicke and Metcalfe (1998) for $H>15.5$. It is also the asymptotic slope of the main belt distribution according to Ivezić et al. (2001). However, according to the latter work, the effective slope in the 15.5-18 range would be steeper (the dashdotted curve in Fig. 1), giving $\gamma \sim 0.3$. In general, if we change the main belt slope from $\gamma^{\prime}$ to $\gamma^{\prime}+\delta \gamma$, the slope of the population captured into resonance passes from $\gamma$ to $\gamma+$ $\delta \gamma$. With this simple rule in mind and assuming the SDSS slope $(\delta \gamma=0.05)$, we can conclude that most of our simulations would give a slope of the NEA population that is too steep even with respect to Stuart's value. But the simulations accounting for YORP, or assuming a very steep size dependence of the reorientation time scale, would give $\gamma \sim 0.39$, compatible with Stuart's NEA population.

This discussion on the slopes assumes that all NEAs come from either the $3 / 1$ or the $\nu_{6}$ resonance, but it should be taken into account that the NEA population is also 
sustained by a network of diffusive resonances (Bottke et al. 2000b, 2002). The bodies transported to the NEA space by chaotic diffusion might have a main-belt-like distribution, unlike those coming through the strong resonances, whose magnitude distribution is steepened by the Yarkovsky effect. Therefore, the slope of the magnitude distribution of the NEA population should be in reality a weighted average of the two slopes. This makes the results of our simulations even more compatible with the observed NEA distribution.

In conclusion, the results of our simulations strongly suggest that the Yarkovsky effect is the major mechanism by which asteroids are continuously supplied to resonances and the NEA population is maintained in steady state. The Yarkovsky effect is compatible-from a quantitative point of view-with both the NEA generation rates and the NEA magnitude distribution. No other mechanism studied so far, including the collisional breakup of parent bodies in the resonance vicinity, are nearly as satisfactory. Our work also shows the importance of the YORP effect for understanding why the NEA magnitude distribution is only moderately steeper than the main belt magnitude distribution, as indicated by the observations. These encouraging results should motivate the development of more sophisticated simulations of the Yarkovsky-driven origin of NEAs. These simulations will need to start from more detailed models of the orbital and magnitude distribution of main belt asteroids, account for a fully consistent collisional cascade process, and take advantage of more accurate estimates of the magnitude of the YORP effect.

\section{Acknowledgments}

We are grateful to David Nesvorný for carefully reading this manuscript and his useful comments. We also acknowledge stimulating discussions with Bill Bottke and Robert Jedicke. We thank the reviewers, D.P. O'Brien and R.S. Gomes, for their valuable suggestions.

\section{References}

Benz, W., Asphaug, E., 1999. Catastrophic disruptions revisited. Icarus $142,5-20$.

Bottke, W.F., Rubincam, D.P., Burns, J.A., 2001a. Dynamical evolution of main belt meteoroids: numerical simulations incorporating planetary perturbations and Yarkovsky thermal forces. Icarus 145, 301-331.

Bottke, W.F., Jedicke, R., Morbidelli, A., Petit, J.M., Gladman, B., 2000b. Understanding the distribution of near-Earth asteroids. Science 288 2190-2194.

Bottke, W.F., Vokrouhlický, D., Brož, M., Nesvorný, D., Morbidelli, A., 2001. Dynamical spreading of asteroid families by the Yarkovsky effect. Science 294, 1694-1696.

Bottke, W.F., Morbidelli, A., Jedicke, R., Petit, J.M., Levison, H., Michel, P., Metcalfe, T.S., 2002. Debiased orbital and magnitude distribution of the near Earth objects. Icarus 156, 399-433.

Bottke, W.F., Vokrouhlický, D., Rubincam, D.P., Brož, M., 2003. Dynamical evolution of asteroids and meteoroids using the Yarkovsky effect, in: Bottke, W.F., Cellino, A., Paolicchi, P., Binzel, R.P. (Eds.), Asteroids III, Univ. of Arizona Press, Tucson, pp. 395-408.

Campo Bagatin, A., Martinez, V.J., Paredes, S., 2002. Multifractal fits to the observed main belt asteroid distribution. Icarus 157, 549-553.

Cellino, A., Michel, P., Tanga, P., Zappalà, V., Paolicchi, P., Dell'Oro, A., 1999. The velocity-size relationship for members of asteroid families and implications for the physics of catastrophic collisions. Icarus 141, 79-95.

Chapman, C.R., Merline, W.J., Thomas, P.C., Joseph, J., Cheng, A.F., Izenberg, N., 2002. Impact history of Eros: craters and boulders. Icarus $155,104-118$.

Dohnanyi, J.W., 1969. Collisional models of asteroids and their debris. J. Geophys. Res. 74, 2531-2554.

Farinella, P., Vokrouhlický, D., 1999. Semimajor axis mobility of asteroidal fragments. Science 283, 1507-1510.

Farinella, P., Gonczi, R., Froeschlé, Ch., Froeschlé, C., 1993. The injection of asteroid fragments into resonance. Icarus 101, 174-187.

Farinella, P., Froeschlé, Ch., Froeschlé, C., Gonczi, R., Hahn, G., Morbidelli, A., Valsecchi, G.B., 1994. Asteroids falling onto the Sun. Nature 371, 315-317.

Farinella, P., Vokrouhlický, D., Hartmann, W.K., 1998. Meteorite delivery via Yarkovsky orbital drift. Icarus 132, 378-387.

Gladman, B., Migliorini, F., Morbidelli, A., Zappalà, V., Michel, P., Cellino, A., Froeschlé, Ch., Levison, H., Bailey, M., Duncan, M., 1997. Dynamical lifetimes of objects injected into asteroid belt resonances. Science 277, 197-201.

Gladman, B., Michel, P., Froeschlé, Ch., 2000. The near-Earth object population. Icarus 146, 176-189.

Greenberg, R., Nolan, M., 1989. Delivery of asteroids and meteorites to the inner Solar System, in: Binzel, R.P., Gehrels, T., Matthew, M.S. (Eds.), Asteroids II, Univ. of Arizona Press, Tueon, pp. 778-904.

Grieve, R.A., Shoemaker, E.M., 1994. The record of past impacts on Earth, in: Gehrels, T., Matthews, M.S. (Eds.), Hazards due to Comets and Asteroids, Univ. of Arizona Press, Tucson, pp. 471-462.

Guillens, S.A., Vieira Martins, R., Gomes, R.S., 2002. A global study of the 3:1 neighborhood: a search for unstable asteroids. Astron. J. 124, 2322-2332.

Ivezić, Z., and 31 coauthors. 2001. Solar System objects observed in the Sloan digital sky survey commissioning data. Astron. J. 122, 27492784.

Jedicke, R., Metcalfe, T.S., 1998. The orbital and absolute magnitude distributions of main belt asteroids. Icarus 131, 245-260.

Jurić, M., and 15 coworkers. 2002. Comparison of asteroids observed in the Sloan digital sky survey with a catalog of known asteroids. Astron. J. 124, 1776-1787.

Knežević, Z., Milani, A., 2000. Synthetic proper elements for outer main belt asteroids. Celest. Mech. Dynam. Astron. 78, 17-46.

Knežević, Z., Lemaitre, A., Milani, A., 2003. Asteroid proper element determination, in: Bottke, W.F., Cellino, A., Paolicchi, P., Binzel, R.P. (Eds.), Asteroids III, Univ. of Arizona Press, Tucson, pp. 603-612.

Love, S.G., Ahrens, T.J., 1996. Catastrophic impacts on gravity dominated asteroids. Icarus 124, 141-155.

Michel, P., Froeschlé, Ch., Farinella, P., 1996. Dynamical evolution of two near-Earth asteroids to be explored by spacecraft: (433) Eros and (4660) Nereus. Astron. Astrophys 313, 993-1007.

Michel, P., Benz, W., Tanga, P., Richardson, D.C., 2001. Collisions and gravitational reaccumulation: forming asteroid families and satellites. Science 294, 1696-1700.

Migliorini, F., Morbidelli, A., Zappalá, V., Gladman, B., Bailey, M., Cellino, A., 1997. Vesta fragments from $\nu_{6}$ and 3:1 resonances: implications for V-type NEAs and HED meteorites. Meteoritics Planet. Sci. 32, 906-913.

Migliorini, F., Michel, P., Morbidelli, A., Nesvorný, D., Zappalà, V., 1998. Origin of multikilometer Earth- and Mars-crossing asteroids: a quantitative simulation. Science 281, 2022-2024.

Morbidelli, A, 2002. Modern Celestial Mechanics Aspects of the Solar System Dynamics. Taylor and Francis, New York. 
Morbidelli, A., Gladman, B., 1998. Orbital and temporal distributions of meteorites originating in the asteroid belt. Meteoritics Planet Sci. 33, 999-1016

Morbidelli, A., Nesvorný, D., 1999. Numerous weak resonances drive asteroids towards terrestrial planets oribts. Icarus 139, 295-308.

Morbidelli, A., Zappalà, V., Moons, M., Cellino, A., Gonczi, R., 1995. Asteroid families close to mean motion resonances: dynamical effects and physical implications. Icarus 118, 132-154.

Morbidelli, A., Bottke, W.F., Froeschlé, Ch., Michel, P., 2002a. Origin and evolution of NEOs, in: Bottke, W.F., Cellino, A., Paolicchi, P., Binzel, R.P. (Eds.), Asteroids III, Univ. of Arizona Press, Tucson, pp. 409422.

Morbidelli, A., Jedicke, R., Bottke, W.F., Michel, P., Tedesco, E.F., 2002b. From magnitudes to diameters: the albedo distribution of near-Earth objects and the Earth collision hazard. Icarus 158, 329342.

Morbidelli, A., Nesvorný, D., Bottke, W.F., Michel, P., Vokrouhlický, D., Tanga, P., 2003. The shallow magnitude distribution of asteroid families. Icarus, in press.

Muinonen, K., Lagerros, J.S.V., 1998. Inversion of shape statistics for small Solar System bodies. Astron. Astrophys. 333, 753-761.

Müller, T.G., Lagerros, J.S.V., 1998. Asteroids as far-infrared photometric standards for ISOPHOT. Astron. Astrophys. 338, 340-352.

Neukum, G., Ivanov, B.A., 1994. Crater size distributions and impact probabilities on Earth from lunar, terrestrial-planet, and asteroid cratering data, in: Gehrels, T., Matthews, M.S., Schumann, A. (Eds.), Hazards due to Comets and Asteroids, Univ. of Arizona Press, Tucson, p. 359.

Nesvorný, D., Morbidelli, A., Vokrouhlický, D., Bottke, W.F., Brož, M., 2002. The Flora family: a case of the dynamically dispersed collisional swarm? Icarus 157, 155-172.

Nesvorný, D., Ferraz-Mello, S., Holman, M., Morbidelli, A., 2003. Regular and chaotic dynamics in the mean motion resonances: implications for the structure and evolution of the asteroid belt, in: Bottke, W.F., Cellino, A., Paolicchi, P., Binzel, R.P. (Ed.), Asteroids III, Univ. of Arizona Press, Tucson, pp. 379-394.

Pravec, P., Harris, A.W., 2000. Fast and slow rotation of asteroids. Icarus 148, 12-20.

Rabinowitz, D.L., 1997. Are main-belt asteroids a sufficient source for the Earth-approaching asteroids? Part I. Predicted vs. observed orbital distributions. Icarus 127, 33-54.
Rabinowitz, D.L., Helin, E., Lawrence, K., Pravdo, S., 2000. A reduced estimate of the number of kilometre-sized near-Earth asteroids. Nature 403, 165-166.

Rubincam, D.P., 2000. Radiative spin-up and spin-down of small asteroids. Icarus $148,2-11$.

Stuart, J.S., 2001. A near-Earth asteroid population estimate from the LINEAR survey. Science 294, 1691-1693.

Tanga, P., Cellino, A., Michel, P., Zappalà, V., Paolicchi, P., Dell'Oro, A., 1999. On the size distribution of asteroid families: the role of geometry. Icarus 141, 65-78.

Tedesco, E.F., Desert, F.X., 2002. The Infrared Space Observatory deep asteroid search. Astron. J. 123, 2070-2082.

Tedesco, E.F., Cellino, A., Zappalá, V., 2002. The statistical asteroid model. I. The main belt population for diameters greater than $1 \mathrm{~km}$. Astron. J., submitted.

Vokrouhlický, D., 1998. Diurnal Yarkovsky effect for meter-sized asteroidal fragments' mobility I. Linear theory. Astron. Astrophys. 335, 1093-1100.

Vokrouhlický, D., 1999. A complete linear model for the Yarkovsky thermal force on spherical asteroid fragments. Astron. Astrophys. 344, 362-366.

Vokrouhlický, D., Čapek, D., 2002. YORP-induced long-term evolution of the spin state of small asteroids and meteoroids. I. Rubincam's approximation. Icarus 159, 449-467.

Vokrouhlický, D., Farinella, P., 2000. Efficient delivery of meteorites to the Earth from a wide range of asteroid parent bodies. Nature 407, 606-608.

Wetherill, G.W., 1979. Steady-state populations of Apollo-Amor objects. Icarus 37, 96-112.

Wetherill, G.W., 1985. Asteroidal source of ordinary chondrites. Meteoritics 20, 1-22.

Wetherill, G.W., 1987. Dynamic relationship between asteroids, meteorites and Apollo-Amor objects. Phil. Trans. R. Soc. London 323, 323-337.

Wetherill, G.W., 1988. Where do the Apollo objects come from? Icarus 76, $1-18$.

Wisdom, J., 1983. Chaotic behaviour and origin of the 3:1 Kirkwood gap. Icarus 56, 51-74

Zappalà, V., Cellino, A., 1996. Main belt asteroids: present and future inventory, in: Completing the Inventory of the Solar System, ASP Conf. Series. 107, 29-44.

Zappalà, V., Cellino, A., Dell'Oro, A., 2002. A search for the collisional parent bodies of large NEAs. Icarus 157, 280-296. 